\title{
One-dimensional theoretical analysis of coupling and confinement effects on the cybotactic clusters of bent-core nematic liquid crystals
}

\author{
Sourav Patranabish, ${ }^{1}$ Yiwei Wang, ${ }^{2}$ Aloka Sinha, ${ }^{1}$ and Apala Majumdar ${ }^{3, *}$ \\ ${ }^{1}$ Department of Physics, Indian Institute of Technology Delhi, Hauz Khas, New Delhi 110016, India \\ ${ }^{2}$ School of Mathematical Sciences, Peking University, Beijing 100871, China \\ ${ }^{3}$ Department of Mathematical Sciences, University of Bath, Bath BA2 7AY, England, United Kingdom
}

(Received 15 July 2018; revised manuscript received 1 October 2018; published 28 January 2019)

\begin{abstract}
The bent-core liquid crystals (LCs) are highly regarded as the next-generation materials for electro-optic devices. The nematic $(\mathrm{N})$ phase of these LCs possesses highly ordered smecticlike cybotactic clusters which are promising for electro-optic applications. We have studied a one-dimensional Landau-de Gennes model of spatially inhomogeneous order parameters for the $\mathrm{N}$ phase of bent-core LCs. We investigate the effects of spatial confinement and coupling (between these clusters and the surrounding LC molecules modeled by a coupling parameter $\gamma$ ) on the order parameters. The coupling is found to increase the cluster order parameter significantly, suggesting enhancement in cluster formation, and also predicts a transition to a phase with weak nematiclike ordering above the nematic supercooling temperature.
\end{abstract}

DOI: 10.1103/PhysRevE.99.012703

\section{INTRODUCTION}

Bent-core liquid crystals (LCs) are regarded as promising materials for the next-generation of electro-optic devices and applications [1,2]. A LC molecule usually has a rodlike shape with a uniaxial cylindrical symmetry [3]. When a bent unit is introduced between two rodlike arms of an LC molecule, a bent-core LC molecule can be realized, but with a reduced symmetry of $C_{2 v}$ type [3]. Bent-core LCs are a recent inclusion in the family of liquid crystals, offering cutting-edge potential applications in LC-based devices [4]. The $\mathrm{N}$ phase of calamitic LCs possesses long-range orientational order, but lacks in any definite positional correlation or ordering. In contrast, the $\mathrm{N}$ phase of bent-core LCs possesses small domains with an additional short-range smecticlike local positional ordering besides the usual long-range orientational order, referred to as cybotactic clusters [2,4-8]. It is believed that the bent-shaped LC molecules, owing to their kinked molecular shape, lock themselves together to form these clusters [9]. The highly debated cybotactic nematic phase $\left(\mathrm{N}_{\mathrm{Cyb}}\right)$ of bent-core LCs has been studied extensively over the past few years and is now established with concrete evidence $[2,10]$. The $\mathrm{N}_{\text {Cyb }}$ phase was initially considered to be a pretransitional effect arising only in the vicinity of the $\mathrm{N}$ to smectic $(\mathrm{Sm})$ phase transition. Now experiments have revealed their existence in the whole $\mathrm{N}$ phase, even in the absence of an underlying Sm phase $[10,11]$. Recent experimental studies show that these short-range Sm-like cybotactic clusters can exist even in the isotropic liquid phase of the bent-shaped compounds $[7,9]$. Studies also show that a strong coupling between the polar order and the director gradients can induce modulated polar phases destabilizing the uniform $\mathrm{N}$ phase of such bend-

*Corresponding author: A.Majumdar@bath.ac.uk shaped molecules (e.g., twist-bend or splay-bend), and can even induce polar blue phases $[12,13]$.

The cybotactic clusters were first realized in the $\mathrm{N}$ phase of 1,2,4-oxadiazole based bent-core LCs using small angle $\mathrm{x}$-ray scattering experiments [14]. Several other experimental techniques (e.g., dynamic light scattering, nuclear magnetic resonance study, dielectric studies, etc.) have also been used by researchers to identify the $\mathrm{N}_{\mathrm{Cyb}}$ phase of bent-core LCs $[7,14,15]$. Recently, the $\mathrm{N}_{\mathrm{Cyb}}$ phase of bent-core LCs has been explored via Cryo-TEM investigations [10]. The results show direct evidence of these clusters, typically in the length scales of 30-50 nm, which are larger than those estimated via the positional correlation lengths. Although verified experimentally, there are relatively few studies [16-19] on the theory of such clusters. Moreover, the theory and the experiments are not always in tandem $[9,19,20]$. While the experiments suggest that these clusters occupy only a small percentage $(\sim 3 \%$ of the LC molecules form clusters) of the whole LC volume $[9,20]$, no conclusive remarks have yet been established by the theoretical studies [16-19].

In a very recent work, Madhusudana has proposed a twostate model for the $\mathrm{N}$ phase of bent-core LCs [19]. He has studied the nematic-isotropic transition of a bent-core LC system using minimization of a Landau-de Gennes-type ( $\mathrm{LdG}$ ) free-energy density. The LdG free-energy density is expressed in terms of two scalar order parameters $S_{c}$ and $S_{g}$, corresponding to the clusters and surrounding LC molecules, respectively. He proposes that less-bent (straightened) excited-state (ES) conformers of bent-core LCs form clusters with smectictype ordering. Using a few simplified assumptions, he predicts the bulk values of the two order parameters $S_{c}$ and $S_{g}$, as a function of temperature and magnetic field energy, at a fixed value of the coupling constant $\gamma$ which is a measure of the coupling between the clusters and the surrounding molecules. His results show that the nematic phase has a moderately high value of the cluster parameter $S_{c}$. At relatively higher 
temperatures the nematic phase undergoes a transition to a paranematic phase (with very small values of the order parameters) and eventually exhibits a weak first-order transition to the isotropic phase at even higher temperatures. The magnetic field dependency shows that $S_{c}$ remains small but nonzero (up to $\sim 0.1$ ) above the nematic-isotropic transition temperature, consistent with the experimental observations that clusters can survive at higher temperatures [7]. Although the model is able to predict clusters, it is limited in the sense that it assumes a fixed number of molecules inside a cluster which is temperature independent and does not include the effects of spatial inhomogeneities in the order parameters, director fields, boundary effects, or the effects of confinement. Experiments, in contrast, suggest that the size and polarity of these clusters strongly depend on the temperature, and also on confinement effects induced by geometry [21]. Therefore, a sizeable amount of theoretical work is still needed to further build on Madhusudana's elegant phenomenological model.

In this paper, we study a one-dimensional (1D) model of spatially inhomogeneous order parameters for the $\mathrm{N}$ phase of bent-core LCs building on the earlier work by Madhusudana [19]. This corresponds to a channel geometry, the depth of which is much smaller than the cross-sectional dimensions, so that it suffices to study a two-dimensional (2D) cross-section. Further, the channel length is assumed to be much larger than the channel width in the $2 \mathrm{D}$ cross-section. Hence, it is reasonable to assume that structural characteristics only depend on one spatial variable across the channel width, justifying a 1D model. We introduce spatial inhomogeneities to the order parameter dependent free-energy density, which allows us to study the effects of boundary conditions and confinement. We use the same notations used by Madhusudana and denote the cluster order parameter as $S_{c}$ and the order parameter for surrounding nematic LCs as $S_{g}$ [19]. We add one-constant elastic energy densities for $S_{c}$ and $S_{g}$ to the free-energy density (to model the spatial inhomogeneities) and the associated Euler-Lagrange equations are numerically solved subject to experimentally motivated boundary conditions. In particular, we model Dirichlet boundary conditions, which necessarily means that both $S_{c}$ and $S_{g}$ are fixed on the boundaries. Boundary conditions can be experimentally controlled, so that the Dirichlet conditions are adequate for certain experimental frameworks [21]. We have particularly focused on the effects of a parameter $\gamma$, which accounts for the coupling between clusters and the surrounding LC molecules, on the cluster order parameter $\left(S_{c}\right)$. In particular, Madhusudana worked with a fixed value of $\gamma$ [19]. We show that larger values of $\gamma$, implying an enhanced coupling between the clusters and the surrounding molecules, boost the cluster order parameter $S_{c}$ in the interior. More specifically, if we use $\gamma=5$ as in [19], then the interior values of $S_{c}$ for a confined system are larger than the values for the spatially homogeneous system studied in [19], i.e., one can speculate that confinement cooperates with the coupling mechanisms to boost interior cluster formation. Experimentally, this suggests a strong core to core interaction (among the aromatic cores) between the constituent LCs and therefore an increase in the associated correlation length [21]. Additionally, we observe that an enhanced coupling also results in enhanced values for $S_{g}$ above the nematic supercooling temperature, compared to, say, $\gamma=0$, i.e., it promotes conventional nematic ordering at higher temperatures. We briefly describe the model and how it compares with the experimental conditions and the mathematical framework in Sec. II. In Sec. III, we discuss the numerical results, and we discuss the main conclusions in Sec. IV. We have also done some preliminary work on extending this work to two dimensions and the results are in good agreement with the $1 \mathrm{D}$ observations.

\section{MODEL}

We consider the nematic phase of bent-core LC molecules in a confined geometry and assume that clusters are formed by straightened (less-bent) conformers of the LC molecules while still being surrounded by others that do not form clusters [19]. We denote the cluster molecules as ES (excited state) and the surrounding ones as GS (ground state) and their associated order parameters as $S_{c}$ and $S_{g}$ respectively, using the same notation as Madhusudana [19]. Under usual experimental conditions, LC molecules are confined in a geometry of thickness, typically in the range of few microns $(\mu \mathrm{m})$, with a definite experimentally imposed molecular alignment at the boundaries (substrate interfaces) [21]. The alignment condition is imposed by coating the two substrate surfaces (glass plates) with a polyimide layer and by gently rubbing the polyimide coated surfaces in a parallel or antiparallel fashion. This rubbing creates grooves on the polyimide layer. The two glass plates are then assembled with their coated layers facing each other in order to create a capillary of desired thickness using spacers. The LC, once introduced to this capillary, is assumed to have a fixed orientation on the surfaces. Therefore, we work with Dirichlet boundary conditions and assume that $S_{c}=0$ (or a fixed constant) at the boundaries. Also, we assume that $S_{g}$ has a constant nonzero value on the boundaries from the imposed orientation condition (aligning layer). The cases for nonzero boundary values of $S_{c}$ are also studied in this paper. We study the LdG-type model for $S_{c}$ and $S_{g}$ proposed by Madhusudana and incorporate one-constant elastic energy densities that account for spatial inhomogeneities in $S_{c}$ and $S_{g}$ [19]. It is important to point out that Madhusudana's free-energy density cannot be derived directly from the Landau-de Gennes free-energy density, largely because the Madhusudana approach is modeling two different kinds of molecules (ES and GS) that couple to each other whereas the Landau-de Gennes free-energy density models a single component, usually rodlike molecules the long axes of which tend to align with each other (see, for example, [22]). The typical Landau-de Gennes free energy, $F_{\mathrm{LG}}[Q]$, is a nonlinear integral functional of a tensor order parameter $Q$ and its spatial derivatives [23,24]. Using a one-constant elastic energy density, the Landau-de Gennes free energy can be written as [22]

$$
F_{\mathrm{LG}}[Q]=\int K|\nabla Q|^{2}(x)+f_{B}[Q(x)] d x,
$$

where $f_{B}$ is the bulk energy density that dictates the bulk nematic-isotropic phase transition as the temperature is lowered, $K$ is the elastic constant (one-constant approximation), and $K|\nabla Q|^{2}$ is the elastic energy density penalizing spatial inhomogeneities. The bulk energy density $f_{B}$ is usually taken 
to be a quartic polynomial in the scalar invariants of $Q$ as written below:

$$
f_{B}(Q)=\frac{\alpha\left(T-T^{*}\right)}{2} \operatorname{tr}\left(Q^{2}\right)-\frac{b}{3} \operatorname{tr}\left(Q^{3}\right)+\frac{c}{4}\left(\operatorname{tr} Q^{2}\right)^{2},
$$

where $\alpha, b$, and $c$ are material-dependent constants [22]. The temperature, $T^{*}$, is the nematic supercooling temperature such that the isotropic phase, $Q=0$, is a local minimizer of the bulk energy (2) for $T>T^{*}$ and ordered uniaxial nematic phases [such as (3) below] are minimizers of (2) for $T<$ $T^{*}$, without accounting for any spatial effects. The closest connection between the two models can be made through the ground-state molecules, i.e., we can define a Landau-de Gennes $Q$ tensor for the ground-state molecules as

$$
Q_{g}=\frac{S_{g}}{2}(3 n \otimes \boldsymbol{n}-I) .
$$

This assumes uniaxial symmetry for the ground-state molecules with order parameter $S_{g}$ and director $\boldsymbol{n}$, which is a unit-vector field that denotes the single distinguished direction of uniaxial orientational order. If we substitute Eq. (3) into Eqs. (1) and (2) above and assume that $\boldsymbol{n}$ is constant, we recover the $S_{g}$-dependent terms in Madhusudana's freeenergy (4) below (up to constants). As mentioned above, we use Madhusudana's free-energy density augmented by oneconstant elastic energy density terms for $S_{c}$ and $S_{g}$ [19]:

$$
\begin{aligned}
F= & K_{g}\left|\nabla S_{g}\right|^{2}+K_{c}\left|\nabla S_{c}\right|^{2}+\left(1-a_{x}\right) \\
& \times\left\{\frac{a_{g}}{2}\left(T-T^{*}\right) S_{g}{ }^{2}-\frac{B_{g}}{3} S_{g}{ }^{3}+\frac{C_{g}}{4} S_{g}{ }^{4}-E_{\mathrm{el}} S_{g}\right\} \\
& +\frac{a_{x}}{N_{c}}\left\{-\left(1-a_{x}\right) \gamma S_{g} S_{c}+\frac{\alpha_{c}}{2} S_{c}{ }^{2}+\frac{\beta_{c}}{4} S_{c}{ }^{4}\right\}-a_{x} J E_{\mathrm{el}} S_{c} .
\end{aligned}
$$

Here, the subscripts $g$ and $c$ denote the GS and the ES molecules, respectively, and the clusters are essentially formed by the ES molecules. $K_{g}$ and $K_{c}$ denote the elastic constants, and $S_{g}$ and $S_{c}$ are the order parameters of the GS molecules and the clusters, respectively. $a_{g}, B_{g}, C_{g}$, and $T^{*}$ are the usual LdG parameters that describe the first-order nematic-isotropic transition for the GS molecules, and $\gamma$ is the coupling parameter between the GS molecules and the clusters [19]. $\alpha_{c}$ and $\beta_{c}$ are coefficients for the saturation terms to ensure that the absolute value of $S_{c}<1$ for at least a certain range of $\gamma . N_{c}$ denotes the number of ES molecules in each cluster. $J$ accounts for the shape anisotropy of ES molecules, $E_{\text {el }}$ is the electric field energy $\left(\frac{1}{2} \varepsilon_{0} \Delta \varepsilon E^{2}\right)$ where $\varepsilon_{0}$ is the free-space permittivity, $\Delta \varepsilon$ is the dielectric anisotropy, $E$ is the applied electric field, and $a_{x}$ is the mole fraction of the ES molecules given by

$$
a_{x}=\exp \left(-E_{\mathrm{ex}} / k_{B} T\right) /\left[1+\exp \left(\frac{-E_{\mathrm{ex}}}{k_{B} T}\right)\right],
$$

where $E_{\text {ex }}$ is the excitation energy of the ES molecules, $k_{B}$ is Boltzmann's constant, and $T$ is the temperature. We take $a_{x}=0.1$ throughout the paper consistent with [19]. The contribution of the surface terms to the total free energy is neglected since we consider strong anchoring modeled by Dirichlet conditions on the boundaries [22].
The corresponding Euler-Lagrange equations can be easily computed to be

$$
\begin{aligned}
2 K_{g} \nabla^{2} S_{g}= & \left(1-a_{x}\right)\left[a_{g}\left(T-T^{*}\right) S_{g}-B_{g} S_{g}{ }^{2}+C_{g} S_{g}{ }^{3}\right. \\
& \left.-E_{\mathrm{el}}-\frac{a_{x} \gamma S_{c}}{N_{c}}\right], \\
2 K_{c} \nabla^{2} S_{c}=a_{x} & {\left[\frac{-\left(1-a_{x}\right) \gamma S_{g}}{N_{c}}+\frac{\alpha_{c} S_{c}}{N_{c}}+\frac{\beta_{c} S_{c}{ }^{3}}{N_{c}}-J E_{\mathrm{el}}\right] . }
\end{aligned}
$$

In one dimension, Eqs. (6) and (7) reduce to

$$
\begin{aligned}
& 2 K_{g} \frac{d^{2} S_{g}}{d x^{2}}=\left(1-a_{x}\right)\left[a_{g}\left(T-T^{*}\right) S_{g}-B_{g} S_{g}{ }^{2}+C_{g} S_{g}{ }^{3}\right. \\
&\left.-E_{\mathrm{el}}-\frac{a_{x} \gamma S_{c}}{N_{c}}\right], \\
& 2 K_{c} \frac{d^{2} S_{c}}{d x^{2}}=a_{x}\left[\frac{-\left(1-a_{x}\right) \gamma S_{g}}{N_{c}}+\frac{\alpha_{c} S_{c}}{N_{c}}+\frac{\beta_{c} S_{c}{ }^{3}}{N_{c}}-J E_{\mathrm{el}}\right],
\end{aligned}
$$

where we assume that $S_{g}$ and $S_{c}$ only vary along the $x$ coordinate. As explained before, this assumption is good for certain thin channel-type geometries. We work with the fixed boundary condition $S_{g}=$ constant (nonzero) and $S_{c}=0$ at both $x=0$ and at $x=D$, where $D$ will be specified later. The cases with nonzero $S_{c}$ at the boundaries are treated separately. This simply describes fixed nematic ordering at the boundaries, consistent with some experimentally reported conditions [21].

\section{Nondimensionalization}

We nondimensionalize the system (8) and (9) for ease of presentation. We use the notations $Y_{1}=S_{g}, Y_{2}=$ $S_{c}, A=\left(1-\mathrm{a}_{\mathrm{x}}\right) a_{g}\left(T-\mathrm{T}^{*}\right), B=\left(1-\mathrm{a}_{\mathrm{x}}\right) B_{g}, C=\left(1-\mathrm{a}_{\mathrm{x}}\right) C_{g}$, $D=a_{x}\left(1-\mathrm{a}_{\mathrm{x}}\right) \gamma / N_{c}, E=\left(1-\mathrm{a}_{\mathrm{x}}\right) E_{\mathrm{el}}, \quad M=\alpha_{c} a_{x} / N_{c}, \quad N=$ $\beta_{c} a_{x} / N_{c}$, and $P=J E_{\mathrm{el}} a_{x}$. Let $\tilde{x}=x / x_{s}, \widetilde{Y}_{1}=Y_{1} / Y_{s}$, and $\widetilde{Y}_{2}=Y_{2} / Y_{s}$ where $x_{s}$ and $Y_{s}$ are the scaling factors. Then Eqs. (8) and (9) reduce to

$$
\begin{gathered}
\frac{d^{2} \widetilde{Y}_{1}}{d \tilde{x}^{2}}=\frac{A x_{s}^{2}}{2 K_{g}} \widetilde{Y}_{1}-\frac{B x_{s}^{2} Y_{s}}{2 K_{g}} \widetilde{Y}_{1}^{2}+\frac{C x_{s}^{2} Y_{s}^{2}}{2 K_{g}} \widetilde{Y}_{1}^{3} \\
-\frac{D x_{s}^{2}}{2 K_{g}} \widetilde{Y}_{2}-\frac{E x_{s}{ }^{2}}{2 K_{g} Y_{s}}, \\
\kappa \frac{d^{2} \widetilde{Y}_{2}}{d \tilde{x}^{2}}=\frac{M x_{s}^{2}}{2 K_{g}} \widetilde{Y}_{2}+\frac{N x_{s}^{2} Y_{s}^{2}}{2 K_{g}} \widetilde{Y}_{2}^{3}-\frac{D x_{s}^{2}}{2 K_{g}} \widetilde{Y}_{1}-\frac{P x_{s}{ }^{2}}{2 K_{g} Y_{s}}
\end{gathered}
$$

where $\kappa=K_{c} / K_{g}=1$ (with the approximation $K_{c}=K_{g}=$ $K)$. We set $\frac{|A| x_{s}{ }^{2}}{2 K_{g}}=1$ and $\frac{B x_{s}{ }^{2} Y_{s}}{2 K_{g}}=1$ so that $x_{s}=\sqrt{2 K_{g} /|A|}$ and $Y_{s}=\frac{|A|}{B}$. For simplicity we let $C_{1}=\frac{C|A|}{B^{2}}, C_{2}=$ $\frac{D}{|A|}, C_{3}=\frac{E B}{|A|^{2}}, C_{4}=\frac{M}{|A|}, C_{5}=\frac{N|A|}{B^{2}}$, and $C_{6}=\frac{P B}{|A|^{2}}$ 
and Eqs. (10) and (11) can be compactly written as

$$
\begin{gathered}
\frac{d^{2} \widetilde{Y}_{1}}{d \tilde{x}^{2}}=\operatorname{sgn}(A) \tilde{Y}_{1}-\widetilde{Y}_{1}^{2}+C_{1} \widetilde{Y}_{1}^{3}-C_{2} \widetilde{Y}_{2}-C_{3}, \\
\frac{d^{2} \widetilde{Y}_{2}}{d \tilde{x}^{2}}=C_{4} \widetilde{Y}_{2}+C_{5} \widetilde{Y}_{2}^{3}-C_{2} \widetilde{Y}_{1}-C_{6},
\end{gathered}
$$

where $\operatorname{sgn}(A)=1$ for $A>0$ and $\operatorname{sgn}(A)=-1$ for $A<0$. We take our domain $\Omega=[0,100]$ or, in dimensional terms, $\Omega$ has length $100 x_{s}$, which is approximately equal to $1 \mu \mathrm{m}$ when $K$ is typically in the range of piconewtons. We impose the following boundary conditions on both boundaries $\tilde{x}=$ 0, 100: (i) $\widetilde{Y}_{1}=1$ and (ii) $\widetilde{Y}_{2}=0$. We do not provide a rigorous justification for the boundary value of $\widetilde{Y}_{1}$ except that it arises naturally from the choice of the scaling factor.

The rescaled free energy is (tilde omitted)

$$
\begin{gathered}
\int_{\Omega} \frac{1}{2} \operatorname{sgn}(A) Y_{1}^{2}-\frac{1}{3} Y_{1}^{3}+\frac{C_{1}}{4} Y_{1}{ }^{4}-C_{3} Y_{1}+\frac{C_{4}}{2} Y_{2}{ }^{2}+\frac{C_{5}}{4} Y_{2}{ }^{4} \\
-C_{6} Y_{2}-C_{2} Y_{1} Y_{2}+\frac{1}{2}\left(\frac{d Y_{1}}{d x}\right)^{2}+\frac{\kappa}{2}\left(\frac{d Y_{2}}{d x}\right)^{2} d x
\end{gathered}
$$

We drop the tildes for brevity and work with $Y_{1}$ and $Y_{2}$ in the rest of the paper (unless stated otherwise), to be interpreted as the rescaled order parameters.

At a fixed temperature $T$ (and therefore at a fixed $a_{x}$ ), the parameter $C_{1}$ is effectively the ratio of the LdG parameters $\left(C_{1} \sim a_{g} C_{g} / B_{g}{ }^{2}\right)$ and remains a constant, and $C_{2}$ is the ratio of coupling constant $\gamma$ and the product of $a_{g}$ and $N_{c}$ (the number of ES molecules in a cluster) $\left(C_{2} \sim \gamma / a_{g} N_{c}\right) . C_{3}$ is proportional to the electric field energy $E_{\mathrm{el}}\left(C_{3} \sim B_{g} E_{\mathrm{el}} / a_{g}{ }^{2}\right)$, $C_{4}$ is the ratio of the cluster LdG parameter $\alpha_{c}$ and the product of $a_{g}$ and $N_{c}\left(C_{4} \sim \alpha_{c} / N_{c} a_{g}\right)$, and $C_{5}$ is the ratio $\sim$ $\beta_{c} a_{g} / B_{g}{ }^{2} N_{c}$ and hence depends only on $N_{c}$. $C_{6}$ accounts for the anisotropy factor $J$ and the electric field energy $E_{\mathrm{el}}\left(C_{6} \sim\right.$ $\left.J E_{\mathrm{el}} B_{g} / a_{g}{ }^{2}\right)$. In our paper, we vary the parameter $C_{2}$, keeping all the other parameters $\left(C_{1}\right.$ and $\left.C_{3}-C_{6}\right)$ fixed, and study the associated effects on $Y_{1}$ and $Y_{2}$. Since we work at a fixed temperature, this only accounts for the variation of the order parameters as a function of the coupling constant $\gamma$.

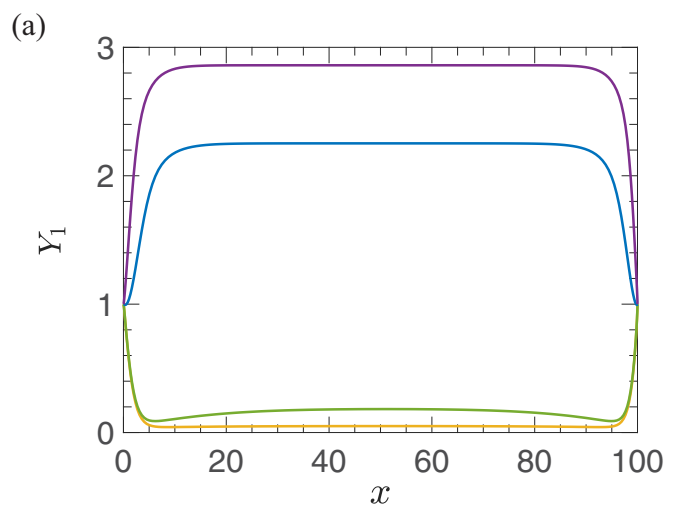

\section{NUMERICAL RESULTS AND DISCUSSION}

We numerically solve Eqs. (12) and (13) to compute $Y_{1}$ and $Y_{2}$ and study their variation with the parameter $C_{2}$, keeping all other parameters $\left(C_{1}\right.$ and $\left.C_{3}-C_{6}\right)$ fixed. The variation of $C_{2}$ only accounts for the influence of coupling constant $\gamma$ on the order parameters at a fixed temperature. We study two representative cases for $A>0$ and $A<0$ separately. Since $A=\left(1-a_{x}\right) a_{g}\left(T-\mathrm{T}^{*}\right)$ and $a_{x}$ is smaller than 1 , for $A>0$ we must have $T>T^{*}$ and for $A<0$ we have $T<T^{*}$. The temperature range $T>T^{*}$ is the so-called high-temperature regime for which the isotropic phase is locally stable for a homogeneous system [when we use the bulk energy (2)] whereas we refer to $T<T^{*}$ as being the low-temperature regime for which the isotropic phase is unstable in the bulk and the bulk energy is minimized by ordered uniaxial states [as in Eq. (3)]. The nondimensionalized 1D domain $\Omega=$ $[0,100]$ (corresponding to $\tilde{x}$ ) is used for the simulations. Since $x=\tilde{x} x_{s}$, and $x_{s} \sim 10^{-8} \mathrm{~m}$ (from the parameters used in our calculations), the physical geometry is in the micron range, consistent with experiments [21]. We have used the following parameter values in our calculations [19]: $K_{g}=$ $K_{c}=K=15 p N=15 \times 10^{-12} N=15 \times 10^{-7}$ dyn (under one constant approximation); $a_{g}=0.04, B_{g}=1.7, C_{g}=$ $4.5, \alpha_{c}=0.22, \beta_{c}=4.0, \quad$ and $\gamma=5.0 \quad\left(a_{g}, B_{g}, C_{g}, \gamma, \alpha_{c}\right.$, and $\beta_{c}$ in $10^{7} / 4 \mathrm{cgs}$ units); $T^{*}=355 \mathrm{~K}, N_{c}=50, J=$ $1.2, E_{\mathrm{el}}=2000 \mathrm{ergs} / \mathrm{cm}^{3}, E_{\mathrm{ex}}=1.1 \times 10^{-13} \mathrm{ergs}$, and $T=$ 360 (for the $A>0$ case) and $350 \mathrm{~K}$ (for the $A<0$ case). This yields $A=0.045 \times 10^{7}, B=0.3825 \times 10^{7}, C=$ $1.0125 \times 10^{7}, D=0.00225 \times 10^{7}, E=1800, M=0.0001$ $\times 10^{7}, N=0.002 \times 10^{7}$, and $P=240$ (in respective cgs units). Therefore we have $C_{1}=0.31142, C_{2}=0.05$ (for $\gamma=$ 5), $C_{3}=0.034, C_{4}=0.00222, C_{5}=0.000615$, and $C_{6}=$ 0.00453 .

The parameter $C_{2}$ is varied in a range between 0.01 and 0.2 corresponding to $\gamma$ in the range 1 to 20. We first discuss the case $A=0.045 \times 10^{7}(T=360 \mathrm{~K})$, i.e., when $T>T^{*}$ with $T-T^{*}=5 \mathrm{~K}$. The numerically computed solutions of $Y_{1}$ and $Y_{2}$ for $A=0.045 \times 10^{7}$ are shown in Fig. 1. The cluster order parameter $Y_{2}$ steadily increases with increasing $C_{2}$ in the bulk and reaches a maximum of $Y_{2} \sim 10$ at $C_{2}=0.2$ (i.e., $\gamma=20) . \gamma$ is a measure of the coupling strength between the clusters and the surrounding LC molecules. Therefore, an (b)

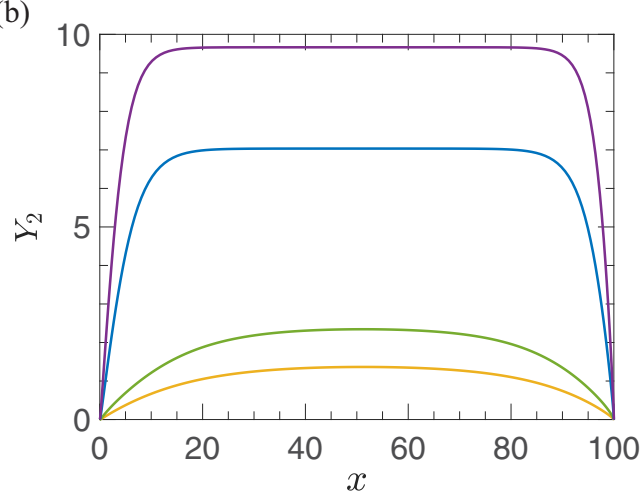

FIG. 1. Numerical results for (a) $Y_{1}$ and (b) $Y_{2}$ in a domain $\Omega=[0,100]$ with $C_{2}=0.01,0.05,0.1$, and 0.2 for $A=0.045 \times 10^{7}>0(T>$ $\left.T^{*}\right)$. The value of $C_{2}$ increases from bottom to top in each figure. 
(a)

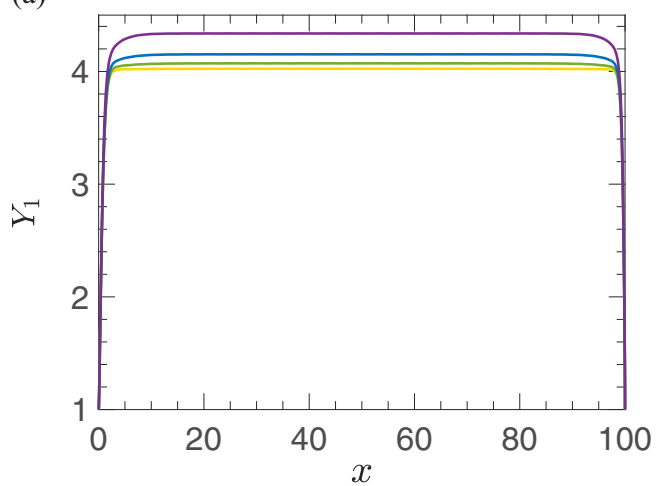

(b)

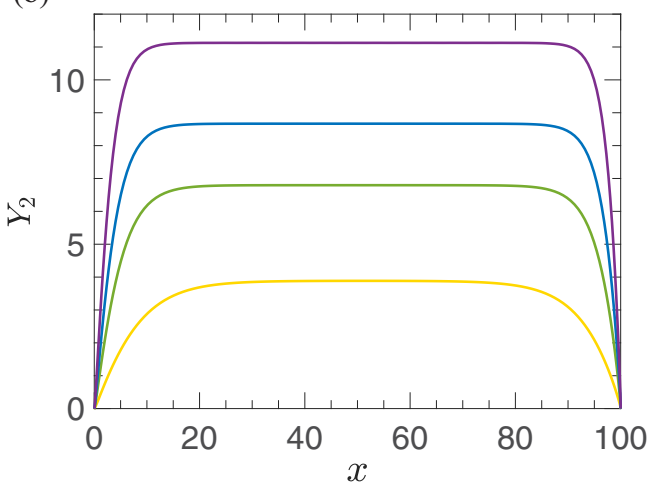

FIG. 2. Numerical results for (a) $Y_{1}$ and (b) $Y_{2}$ in a domain $\Omega=[0,100]$ with $C_{2}=0.01,0.05,0.1$, and 0.2 for $A=-0.045 \times 10^{7}<$ $0\left(T<T^{*}\right)$. The value of $C_{2}$ increases from bottom to top in each figure.

enhanced $\gamma$ is expected to enhance the cluster order parameter $\left(Y_{2}\right)$ as it suggests a very strong core to core interaction (among the aromatic cores) between the constituent LCs and hence an increase in the associated correlation length [21]. The solutions to $Y_{1}$ also exhibit an appreciable increase with increasing $C_{2}$ but with a distinct jump between $C_{2}=0.05$ and 0.1 (at $C_{2} \approx 0.07$ to be precise), indicating a transition to a more ordered phase above the nematic supercooling temperature $T^{*}$, induced by the enhanced coupling. Therefore, an enhanced $\gamma$ not only induces a strong tendency to form clusters but also invokes a stronger ordering in the surrounding molecules. This observation is qualitatively similar to that of the intermediate coupling-induced paranematic phase reported by Madhusudana, who works with $\gamma=5[19,25]$. However, the values of $Y_{1}$ are quite small compared to those of $Y_{2}$, suggesting that clusters may also exist in relatively disordered ambient phases above the supercooling temperature $T^{*}$. Experimentally, the stronger coupling may be realized by enhancing the transverse dipole moment of the LC molecules, which will enhance the core to core interaction and therefore the formation of clusters [26].

When $A=-0.045 \times 10^{7}(T=350 \mathrm{~K})$, the system is well within the nematic phase according to the Landau-de Gennes theory. The numerical solutions for $Y_{1}$ and $Y_{2}$ at $A=$ $-0.045 \times 10^{7}$ are shown in Fig. 2. As expected, the distinct jump in $Y_{1}$ with increasing $C_{2}$ is not observed since the nematic ordering in the ground-state molecules is well settled. Only small changes in the $Y_{1}$ profile are observed with increasing $C_{2}$, which shows that in the low-temperature regime the nematic order of the GS molecules is largely unaltered by the enhanced coupling. In contrast, the cluster parameter $Y_{2}$ is observed to change significantly with $C_{2}$ and thus with $\gamma$. This behavior is similar to the case $A=0.045 \times 10^{7}$, but the values of $Y_{2}$ are comparatively larger for $A=-0.045 \times$ $10^{7}\left(T<T^{*}\right)$. This is again in line with the melting of clusters at higher temperatures [19]. Experimentally we have also observed similar signatures of reduction in cluster size with increasing temperature in one of our earlier works on bentcore systems [21]. Moreover, the $Y_{2}$ profile, which is parabolic at smaller $(\sim 0.05) \gamma$ values (implying the presence of clusters near the bulk center), becomes more flat and plateaulike at higher $(\sim 0.1-0.2) \gamma$ values, suggesting that the clusters are evenly distributed in the bulk. It is interesting to note that the value of $Y_{1}$ for $A>0$ (when $T>T^{*}$ ) in the case of an enhanced $\gamma$ is comparable to $Y_{1}$ for $A<0$ for smaller values of $\gamma$ (in the nematic phase), supporting our claim that phases with nematiclike ordering (similar to the coupling-induced paranematic phase) are stabilized by enhanced coupling above the nematic supercooling temperature.

We comment on bounds for the order parameters $S_{g}$ and $S_{c}$ in the Madhusudana free-energy [see Eq. (4)]. Both $S_{g}$ and $S_{c}$ should be bounded by unity by their statistical definition, i.e., $Y_{i} \leqslant B /|A|$ in the nondimensionalized sense. However, the numerical results in Figs. 1 and 2 do not always satisfy this statistical bound. In [27], the author studies bounds on the nematic scalar order parameter (analogous to $S_{g}$ ) for spatially inhomogeneous systems within the classical Landau-de Gennes framework described by Eq. (1) and finds that the order parameter (for physically relevant equilibria) can be greater than unity. This is because the Landau-de Gennes free-energy density is phenomenological based on a Taylor expansion near the isotropic phase, $Q=0$, and is not derived from the statistical mechanical interpretation of $Q$ as being the second moment of a probability distribution function for the molecular orientations. Hence, strictly speaking, the classical Landau-de Gennes theory is only valid near the isotropic-nematic transition temperature. There are no natural bounds on the nematic scalar order parameter in the Landau-de Gennes framework in general. When the scalar order parameter has nonphysical values greater than unity,

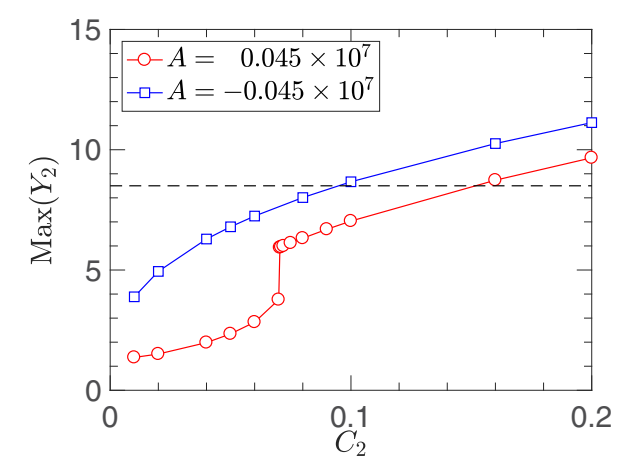

FIG. 3. Upper bound for $Y_{2}$ as a function of $C_{2}$ for both $A=0.045 \times 10^{7}(>0)$ and $-0.045 \times 10^{7}(<0)$. The dashed line corresponds to $Y_{2}=B /|A|=8.5$, or equivalently $S_{c}=1$. 

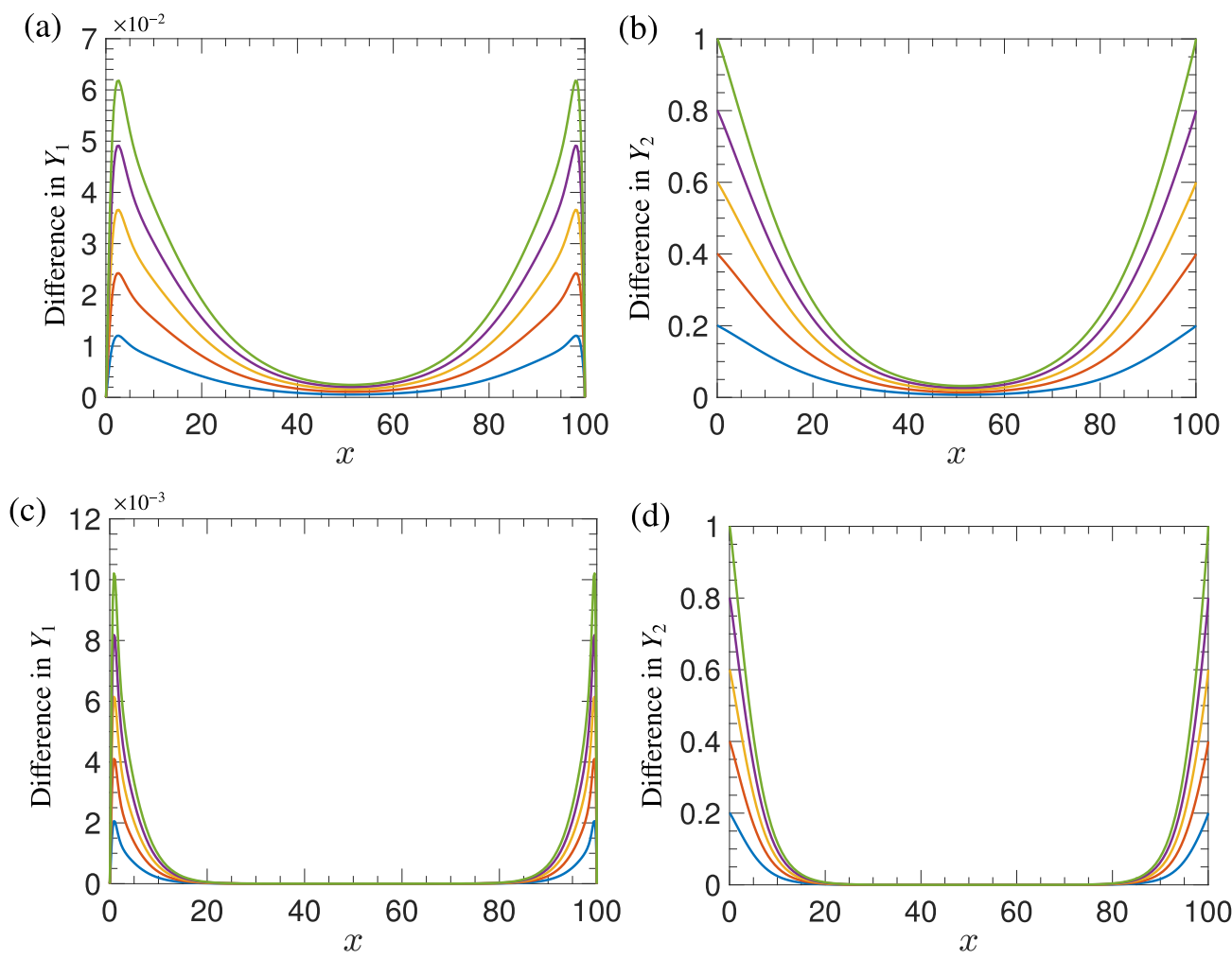

FIG. 4. Differences between solutions with nonzero boundary condition $\left(Y_{2}=0.2,0.4,0.6,0.8\right.$, and 1.0$)$ and zero boundary condition $\left(Y_{2}=0\right)$ when $C_{2}=0.05$ ( $Y_{1}$, left column; $Y_{2}$, right column). Results for $A=0.045 \times 10^{7}(T=360 \mathrm{~K})$ are shown in the first row (a, b), while results for $A=-0.045 \times 10^{7}(T=350 \mathrm{~K})$ are shown in the second row (c, d). The value of nonzero $Y_{2}$ imposed on the boundary increases from bottom to top in each figure.

e.g., for low temperatures, higher-order terms have to be included into the bulk energy or singular bulk energies can be employed. In a similar vein, Madhusudana's free-energy density is a phenomenological approach and is not derived from statistical mechanical interpretations of $S_{g}$ and $S_{c}$ as being related to the second moments of appropriately defined probability distribution functions. Hence, there is no reason why this model would predict values less than unity for $S_{g}$ and $S_{c}$; we can interpret the regime for which $\max \left(S_{g}\right)$ and $\max \left(S_{c}\right)$ are less than or equal to unity as being the physically relevant regime $\left[\min \left(S_{g}\right)\right.$ and $\min \left(S_{c}\right)$ are always within the physically relevant regime according to the numerical results].

To identify the regime where $Y_{i} \leqslant B /|A|$ (i.e., $S_{i} \leqslant 1$ ) always holds, we compute the maximum value of $Y_{2}$ as a function of $C_{2}$ for the cases $A=-0.045 \times 10^{7}$ and $0.045 \times$ $10^{7}$. The variation of $\max \left(Y_{2}\right)$ with $C_{2}$ is shown in Fig. 3. Both the profiles show a continuous increase in $Y_{2}$ with $C_{2}$ due to the coupling-induced ordering except for a jump at $C_{2} \approx 0.07$ (i.e., $\gamma \approx 7$ ) for $A=0.045 \times 10^{7}$. The physical bound on $\max \left(Y_{2}\right)$ is shown by the dashed line in Fig. 3, which then gives the corresponding upper bound for $C_{2}$. For $A=-0.045 \times 10^{7}$ the bound is $C_{2} \leqslant 0.1$, while for $A>$ 0 the bound is $C_{2} \leqslant 0.16$. Therefore, we conjecture that $\gamma<10$ for physically relevant solutions when $T=350 \mathrm{~K}$ (low-temperature regime) and $\gamma<16$ for physically relevant solutions when $T=360 \mathrm{~K}$ (high-temperature regime).

For completeness, we also performed simulations with nonzero $Y_{2}$ at the boundaries for $A=0.045 \times 10^{7}$ and $-0.045 \times 10^{7}$. The difference between these solutions and the solution with $Y_{2}=0$ at the boundary are evaluated and shown in Fig. 4 when $C_{2}=0.05$. It can be noticed that the boundary conditions really only govern the boundary layers while the interior values are only heavily influenced by the coupling constant $C_{2}$. For $A=-0.045 \times 10^{7}$, when the boundary layers are sharp, as indicated in Fig. 4, the bulk values are almost the same for different boundary conditions. For $A=0.045 \times$ $10^{7}$, the relative differences between solutions with $Y_{2}=1$ and $Y_{2}=0$ at the boundaries, respectively, are only about $1 \%$.

\section{Preliminary work in two dimensions}

In two dimensions, for $A=0.045 \times 10^{7}$ the EulerLagrange equations (12) and (13) are

$$
\begin{gathered}
\frac{\partial^{2} \widetilde{Y}_{1}}{\partial \tilde{x}^{2}}+\frac{\partial^{2} \widetilde{Y}_{1}}{\partial \tilde{y}^{2}}=\widetilde{Y}_{1}-\widetilde{Y}_{1}^{2}+C_{1} \widetilde{Y}_{1}^{3}-C_{2} \widetilde{Y}_{2}-C_{3}, \\
\frac{\partial^{2} \widetilde{Y}_{2}}{\partial \tilde{x}^{2}}+\frac{\partial^{2} \widetilde{Y}_{2}}{\partial \tilde{y}^{2}}=C_{4} \widetilde{Y}_{2}+C_{5} \widetilde{Y}_{2}^{3}-C_{2} \widetilde{Y}_{1}-C_{6} .
\end{gathered}
$$

We now solve Eqs. (15) and (16) in a nondimensionalized 2D domain $\Sigma=[0,100: 0,100]$ with the boundary conditions (tilde omitted) (i) $Y_{1}=1$ and (ii) $Y_{2}=0$ on all four boundaries. The COMSOL [28] generated plots for $Y_{1}$ and $Y_{2}$ when $C_{2}=0.01$ and 0.05 are shown in Fig. 5 (in tilde omitted notation). It can be observed that the increment of $C_{2}$ has a similar effect on $Y_{2}$ in two dimensions with very little effect on $Y_{1}$ (as in one dimension). Therefore, the qualitative conclusions remain the same in one and two dimensions. 

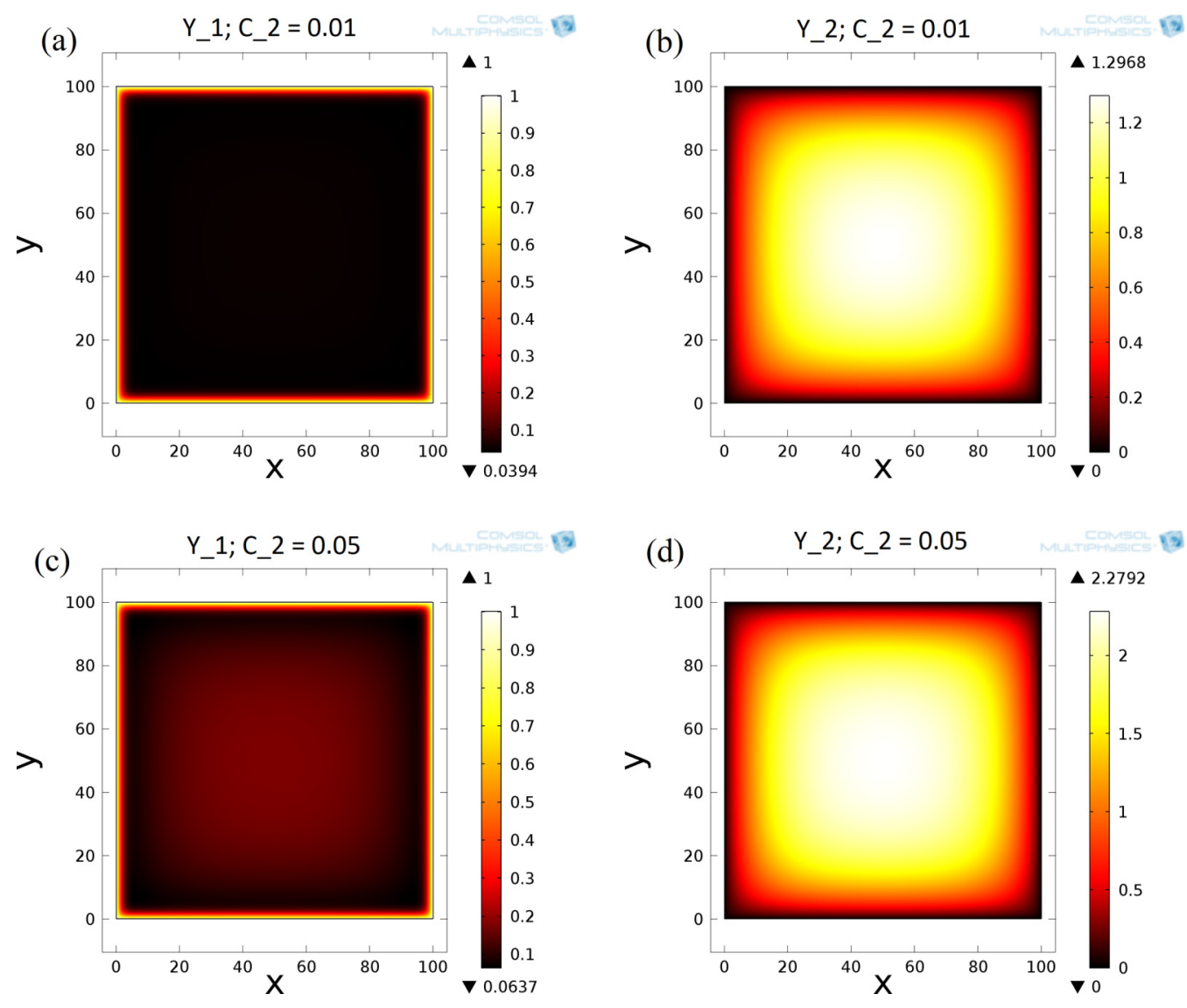

FIG. 5. COMSOL generated solutions of (a, c) $Y_{1}$ and (b, d) $Y_{2}$ for $A=0.045 \times 10^{7}$ in a 2D domain $\Sigma=[0,100: 0,100]$ when $C_{2}=0.01$ (a, b) and $0.05(\mathrm{c}, \mathrm{d})$.

\section{CONCLUSION}

We have studied a 1D model of bent-core nematic LCs in a confinement, using a free energy proposed by Madhusudana with the additional effects of spatial inhomogeneities on the order parameters $S_{c}$ and $S_{g}$. The results are discussed in terms of the rescaled order parameters $Y_{1}$ and $Y_{2}$, representative of the actual order parameters $S_{g}$ and $S_{c}$, respectively. We work with Dirichlet boundary conditions and assume $\left[Y_{1}, Y_{2}\right]=$ $[1,0]$ on the boundaries. We also study nonzero boundary conditions for $Y_{2}$, which do not significantly change the order parameters in the interior of the domain. Our numerical results show the effects of coupling $(\gamma)$ between the clusters and the surrounding LC molecules in the low-temperature and high-temperature regimes. Increasing $\gamma$ boosts the cluster order parameter $\left(Y_{2}\right.$ and hence $\left.S_{c}\right)$ and therefore suggests enhancement in cluster formation. Experimentally, this can be related to the increased core to core interaction between the bent-core mesogens [21]. This promotes the formation of smecticlike highly ordered cybotactic clusters in the lowtemperature regime and even extends into higher temperatures (since we observe finite values of $Y_{2}$ for moderate coupling when $T=360 \mathrm{~K}$ ). The $Y_{2}$ profile is parabolic at smaller $\gamma$ values and becomes more flat and plateaulike when $\gamma$ is quite high $(\sim 0.1-0.2)$, suggesting an even distribution of clusters in the bulk. Compared to the spatially homogeneous study by Madhusudana at $\gamma=5$, we observe an enhanced $S_{c}\left(=Y_{2} \times \frac{|A|}{B}\right)$ for $T<T^{*}[19]$. For $\gamma=5$ and $T=350 \mathrm{~K}$, we obtain $S_{c} \sim 0.8$ in the interior whereas Madhusudana reports $S_{c} \sim 0.6$ without confinement effects and we observe interior values of $S_{c} \sim 0.3$ for $T=360 \mathrm{~K}$. For $C_{2} \geqslant 0.07$ (i.e., $\gamma \geqslant 7$ ), the interior value of $S_{c}$ reaches $\sim 0.9$ for $T=$ $350 \mathrm{~K}$. Further, $\gamma$ also has a profound effect on the order parameter $Y_{1}$ (and hence $S_{g}$ ) of the surrounding molecules and induces a transition to a phase with weak nematiclike ordering when $T=360 \mathrm{~K}$. However, in the low-temperature regime ( $T=350 \mathrm{~K})$, the effect of $\gamma$ on $Y_{1}$ is not significant. We also briefly consider the effect of nonzero boundary conditions for $Y_{2}$. The differences between the numerical solutions in the interior, computed with $Y_{2}=0$ and nonzero values of $Y_{2}$ on the boundaries, are not appreciable. We conjecture that the interior profiles are largely dictated by the coupling between the ES and GS molecules and how they align with respect to one another to minimize their elastic distortion energies and the bulk coupling energies in Eq. (4).

Future work includes the incorporation of weak anchoring or surface energies into this free-energy based approach for bent-core systems along with position-dependent volume fractions of clusters, modeled by the parameter $a_{x}$ in Eq. (4), which might be relevant for inhomogeneous systems. For simplicity, we have worked with a fixed value of $a_{x}$ in this paper, since we work at fixed temperatures, and the boundary conditions are motivated by experimental considerations. However, the spatial dependence of $a_{x}$ and possibly other variables will be more important for systems with weak anchoring, for 
which the boundary conditions are not explicitly prescribed. This will be reported in future work.

\section{ACKNOWLEDGMENTS}

S.P. acknowledges IIT Delhi for financial support under Full-time Institute Assistantship. A.M. acknowledges support from an OCIAM Visiting Fellowship and the Keble Advanced
Studies Centre. The authors would like to thank DST-UKIERI for generous funding to support the 3-year collaborative project. The authors gratefully acknowledge IIT Delhi for the COMSOL platform and for the computational resources. S.P. and A.S. thank the Department of Mathematical Sciences for their hospitality during their research visits. S.P. thanks Professor Paul Milewski for discussions on the numerical simulations.
[1] J. Etxebarria and M. Blanca Ros, J. Mater. Chem. 18, 2919 (2008).

[2] O. Francescangeli, F. Vita, and E. T. Samulski, Soft Matter 10, 7685 (2014).

[3] H. Takezoe and Y. Takanishi, Jpn. J. Appl. Phys. 45, 597 (2006).

[4] A. Jákli, Liq. Cryst. Rev. 1, 65 (2013).

[5] G. Shanker, M. Prehm, M. Nagaraj, J. K. Vij, M. Weyland, A. Eremin, and C. Tschierske, Chem. Phys. Chem. 15, 1323 (2014).

[6] G. Shanker, M. Nagaraj, A. Kocot, J. K. Vij, M. Prehm, and C. Tschierske, Adv. Funct. Mater. 22, 1671 (2012).

[7] S. Ghosh, N. Begum, S. Turlapati, S. K. Roy, A. K. Das, and N. V. S. Rao, J. Mater. Chem. C 2, 425 (2014).

[8] O. Francescangeli, V. Stanic, S. I. Torgova, A. Strigazzi, N. Scaramuzza, C. Ferrero, I. P. Dolbnya, T. M. Weiss, R. Berardi, L. Muccioli, S. Orlandi, and C. Zannoni, Adv. Funct. Mater. 19, 2592 (2009).

[9] C. Bailey, K. Fodor-Csorba, J. T. Gleeson, S. N. Sprunt, and A. Jákli, Soft Matter 5, 3618 (2009).

[10] C. Zhang, M. Gao, N. Diorio, W. Weissflog, U. Baumeister, S. Sprunt, J. T. Gleeson, and A. Jákli, Phys. Rev. Lett. 109, 107802 (2012).

[11] A. De Vries, Mol. Cryst. Liq. Cryst. 10, 219 (1970).

[12] S. M. Shamid, S. Dhakal, and J. V. Selinger, Phys. Rev. E 87, 052503 (2013).

[13] S. M. Shamid, D. W. Allender, and J. V. Selinger, Phys. Rev. Lett. 113, 237801 (2014).
[14] V. Domenici, C. A. Veracini, and B. Zalar, Soft Matter 1, 408 (2005).

[15] S. Stojadinovic, A. Adorjan, S. Sprunt, H. Sawade, and A. Jakli, Phys. Rev. E 66, 060701(R) (2002).

[16] A. G. Vanakaras and D. J. Photinos, J. Chem. Phys. 128, 1 (2008).

[17] S. Droulias, A. G. Vanakaras, and D. J. Photinos, Liq. Cryst. 37, 969 (2010).

[18] S. D. Peroukidis, A. G. Vanakaras, and D. J. Photinos, Phys. Rev. E 84, 010702(R) (2011).

[19] N. V. Madhusudana, Phys. Rev. E 96, 022710 (2017).

[20] C. Bailey, K. Fodor-Csorba, R. Verduzco, J. T. Gleeson, S. Sprunt, and A. Jákli, Phys. Rev. Lett. 103, 237803 (2009).

[21] S. Patranabish, G. Mohiuddin, N. Begum, A. R. Laskar, S. K. Pal, N. V. S. Rao, and A. Sinha, J. Mol. Liq. 257, 144 (2018).

[22] P. G. de Gennes and J. Prost, The Physics of Liquid Crystals (Oxford University Press, Oxford, 1993).

[23] A. Majumdar and A. Zarnescu, Arch. Ration. Mech. Anal. 196, 227 (2010).

[24] E. G. Virga, Variational Theories for Liquid Crystals (CRC Press, Boca Raton, 1995).

[25] T. Ostapenko, D. B. Wiant, S. N. Sprunt, A. Jákli, and J. T. Gleeson, Phys. Rev. Lett. 101, 247801 (2008).

[26] C. Tschierske and D. J. Photinos, J. Mater. Chem. 20, 4263 (2010).

[27] A. Majumdar, Eur. J. Appl. Math. 21, 181 (2010).

[28] COMSOL Multiphysics version 4.3, COMSOL AB, Stockholm, Sweden, www.comsol.com. 